\title{
Some insults are easier to detect: the embodied insult detection effect
}

\author{
Michele Wellsby ${ }^{1}$, Paul D. Siakaluk ${ }^{1 *}$, Penny M. Pexman ${ }^{2}$ and William J. Owen ${ }^{1}$ \\ 1 Psychology Department, University of Northern British Columbia, Prince George, BC, Canada \\ 2 Department of Psychology, University of Calgary, Calgary, AB, Canada
}

\section{Edited by:}

Diane Pecher, Erasmus University

Rotterdam, Netherlands

\section{Reviewed by:}

Michael Kaschak, Florida State

University, USA

Shirley-Ann Rueschemeyer, Radboud

University Nijmegen, Netherlands

*Correspondence:

Paul D. Siakaluk, Psychology

Department, University of Northern

British Columbia, Prince George, BC,

Canada V2N $4 Z 9$.

e-mail:siakaluk@unbc.ca
In the present research we examined the effects of bodily experience on processing of insults in a series of semantic categorization tasks we call insult detection tasks (i.e., participants decided whether presented stimuli were insults or not). Two types of insults were used: more embodied insults (e.g., asswipe, ug/y), and less embodied insults (e.g., cheapskate, twit), as well as non-insults. In Experiments 1 and 2 the non-insults did not form a single, coherent category (e.g., airbase, polka), whereas in Experiment 3 all the non-insults were compliments (e.g., eyeful, honest). Regardless of type of non-insult used, we observed facilitatory embodied insult effects such that more embodied insults were responded to faster and recalled more often than less embodied insults. In Experiment 4 we used a larger set of insults as stimuli, which allowed hierarchical multiple regression analyses. These analyses revealed that bodily experience ratings accounted for a significant amount of unique response latency, response error, and recall variability for responses to insults, even with several other predictor variables (e.g., frequency, offensiveness, imageability) included in the analyses: responses were faster and more accurate, and there was greater recall for relatively more embodied insults. These results demonstrate that conceptual knowledge of insults is grounded in knowledge gained through bodily experience.

Keywords: embodied cognition, insult processing, conceptual processing, mental simulation
The emerging viewpoint of embodied cognition holds that cognitive processes are deeply rooted in bodily interactions with the environment (Wilson, 2002). That is, bodily interactions with the environment are integral to the acquisition of knowledge and to the development of cognitive processes that bear on that knowledge (Barsalou, 1999). The embodied cognition viewpoint contrasts with the long held classical cognitive viewpoint (known as cognitivism), which claims that cognitive processes are not related to bodily interactions with the environment; rather, cognitive processes are proposed to be non-embodied in the sense that they are independent from knowledge gained from bodily experience. As such, by the cognitivist account, cognitive processing involves the manipulation of abstract symbols via rules in the mind (Barsalou, 1999; Cowart, 2004).

Barsalou (1999) developed an embodied cognition account called perceptual symbol systems theory. According to this theory, bodily interactions with the environment are crucial to many cognitive processes. He suggested that the modality-specific neural systems used for perception and action are also used to represent concepts in the brain, through the process of simulation. In other words, conceptual processing is, in a fundamental way, grounded in neural systems involved in perceptual and motor processing. More specifically, simulation is the partial neural re-enactment of perceptual, motor, and introspective states acquired during bodily experiences with the environment (Barsalou, 2008). For example, when we have bodily experience interacting with a particular car, the brain captures states across all sensory, motor, and introspective modalities (e.g., what the car looks and smells like, the bodily "feel" of getting into and driving the car, the fear that may result from nearly getting into an accident, etc.) and integrates them into a multimodal neural representation stored in memory. Later, when knowledge is needed to represent the category $C A R$, these multimodal neural representations are partially reactivated to simulate how the brain represented the original experiences. In other words, off-line cognition (i.e., cognizing about an object or event that is not currently present) is body-based (Wilson, 2002): When we think about an object or event, we are neurally re-experiencing the sensory, motor, and introspective components associated with that object or event from previous bodily experience.

The notion of simulation has been invoked to explain recent demonstrations that knowledge gained through bodily experience influences linguistic processing. The literature on sentence processing contains several such examples, including the action-sentence compatibility effect (Glenberg and Kaschak, 2002; Glenberg et al., 2008) and what we will call the visibility-sentence compatibility effect (Yaxley and Zwaan, 2007).

Using a sentence verification task (i.e., does the sentence make sense?), Glenberg et al. (2008) reported an interaction between type of sentence (whether it referred to something being transferred toward or away from one's body) and type of response (whether it required moving one's hand toward or away from one's body), such that responses to toward sentences were faster when they required a toward response as compared to an away response, and responses to away sentences were faster when they required an away response as compared to a toward response. Interestingly, this action-sentence compatibility effect was observed for sentences that described 
transfer of either concrete objects (e.g., papers) or abstract concepts (e.g., responsibilities). Glenberg et al. explained their findings in the following way. When participants read a sentence that involved something being transferred away from them, for example, they created a simulation of either the concrete object or abstract concept going away from them. They were faster at making a button press that required moving their hand away from their bodies because the away action was congruent with the direction of transfer in the simulation. Conversely, in the away condition, participants were slower at making a button press that required moving their hand toward their bodies, because this toward action was incongruent with the direction of transfer in the simulation.

Using a sentence-picture verification task (i.e., participants read a sentence and then verified if a pictured object was in that sentence), Yaxley and Zwaan (2007) reported an interaction between type of visibility described in a sentence (clear or unclear) and type of picture resolution (clear or unclear). An example of a clear sentence is "Through the clean goggles, the skier could easily identify the moose," and an example of an unclear sentence is "Through the fogged goggles, the skier could hardly identify the moose." The clear pictures were presented at $100 \%$ resolution, whereas the unclear pictures were presented at $50 \%$ resolution (pictures in this condition resembled a snowy picture on television). Yaxley and Zwaan reported that when participants read a clear sentence, clear pictures were verified faster than unclear pictures. Remarkably, when participants read an unclear sentence, unclear pictures were verified faster than clear pictures. Yaxley and Zwaan proposed that when participants read the sentences, the sentences were understood by creating simulations of implied perceptual visibility; thus, when reading clear sentences, participants simulated an unobstructed viewpoint, and could, for example, simulate clearly seeing a moose. When the participants then saw a clear picture of a moose, this matched the simulation they had created, and response latencies were faster as compared to the unclearly presented picture of the moose, which was incongruent with the simulation. Alternatively, when participants read unclear sentences, they simulated an obstructed view, where they could barely make out a moose. When they then saw an unclear picture of a moose, this matched the simulation they had created, and responses were faster to the unclear picture as compared to the clear picture.

A second area of linguistic processing where embodied knowledge is demonstrated to have an influence is in the understanding of metaphors. Neisser (2003) stated that metaphoric thought is an act of imagination, which is mediated by human embodiment. Lakoff and Johnson (1999) also proposed that abstract concepts are grounded metaphorically in embodied knowledge. They argued that people possess extensive knowledge about their bodies and environments, and that abstract concepts draw on this knowledge metaphorically. One example Lakoff and Johnson used to explain the role that bodily experience plays in the comprehension of metaphors is the metaphor BAD IS STINKY. When someone says, "That book stinks!" we understand that she is stating her belief that the content of the book is bad, and not referring to the actual smell of the book. Our understanding of this metaphor, according to Lakoff and Johnson, is mediated by the sensory domain of smell and our having the primary experience of being repelled by foul-smelling objects. We can use this knowledge to make an evaluation about something abstract, such as the content of a book.
Wilson and Gibbs (2007) recently demonstrated how bodily knowledge facilitates conceptual processing of abstract metaphors. They examined the role of simulation in the understanding of abstract metaphors for which the associated expressions are impossible to physically perform, such as swallow your pride or push the argument. Similar to Lakoff and Johnson (1999), they reasoned that simulations for abstract metaphors are based on sensorimotor knowledge gained through physically interacting with objects, such as swallow your food and push the carriage. They reported that abstract metaphors were understood faster when participants made or imagined making a congruent body movement just before reading a metaphorical phrase (e.g., making or imagining making a pushing movement just before reading push the argument), than when they either made or imagined making an incongruent body movement (e.g., making or imagining making a swallowing movement just before reading push the argument), or did not make or imagine making any body movement (i.e., they simply read the metaphorical phrase).

A third area of linguistic processing in which effects of embodied knowledge have been demonstrated is word recognition. One example is the object manipulation effect reported by Myung et al. (2006). Myung et al. used an auditory lexical decision task in which they presented primes that were either related or unrelated to targets, where relatedness was defined by overlap in manipulation features. For example, the prime typewriter would be related to the target piano because they share common manipulation features (i.e., using both hands, with fingers in a curled position that press downward), whereas the prime typewriter would not be related to the target screwdriver because they have different manipulation features (unlike typewriter, screwdriver has the manipulation features of using one hand, with fingers in a grasping position, and twisting of the wrist). Myung et al. reported that responses were faster when targets shared manipulation features with their primes (e.g., typewriter-piano versus typewriter-screwdriver), and proposed the following explanation for their results. When participants heard the prime, they simulated the type of physical manipulation associated with the object. For example, when participants heard the prime typewriter, they simulated how a human would physically manipulate a typewriter (i.e., fingers curled and pressing downward). When participants then had to decide whether piano was a real word or not, they were quick to decide that the required response was "yes," because the simulation they associated with typewriter was similar to the simulation they associated with piano. However, when participants heard the prime word typewriter and then had to decide whether screwdriver was a real word or not, the responses were slower, because the simulation of manipulating a typewriter differs from the simulation of manipulating a screwdriver.

A second example of embodied knowledge influencing word recognition is the body-object interaction (BOI) effect. BOI is a variable that assesses the ease with which a human body can physically interact with a word's referent. Facilitatory BOI effects (i.e., faster responding to words rated high in BOI, such as mask, than to words rated low in BOI, such as ship) have been reported in visual lexical decision and phonological lexical decision tasks (Siakaluk et al., 2008a; Tillotson et al., 2008) and in semantic categorization tasks (Siakaluk et al., 2008b; Wellsby et al., in press). The BOI effect 
is consistent with the notion that words rated high in BOI elicit richer motor simulations of how human bodies physically interact with their referents, and that these richer motor simulations allow for more efficient responding to the words that refer to them. Taken together, the BOI effect and the object manipulation effect suggest that knowledge based on bodily experience is an important part of the semantic representations of words.

In the word recognition research reviewed above (i.e., research examining object manipulation and BOI effects), the stimuli of interest were all concrete concepts; that is, the words used referred to concrete entities like piano, typewriter, mask, and ship. There has been much less research on the role of bodily experience in the processing of words referring to more abstract concepts; that is, words for which the referents or meanings are less tangible. Indeed, one aim of this special issue was to address processing of abstract concepts. In the present research we approached this subject by examining processing of a particular kind of abstract meaning; that is, the negative inferences conveyed by insults.

An insult is a verbal expression that conveys a negative (e.g., offensive, degrading) meaning. Many insults, such as stupid and $u g l y$, are abstract in the sense that they express a quality or attribute distinct from any particular person. That is, they qualify people in a negative manner (e.g., she is stupid or he is ugly). This way of conceptualizing the idea of abstractness as separable from any specific object or instance is what is often meant when cognitive scientists talk about something being abstract. However, there are other ways of conceptualizing the abstractness of insults. One such way is the use of insults, such as lunatic and asswipe, in a metaphorical manner. That is, knowledge gained from one context (e.g., general semantic knowledge, bodily experience) is applied to a different context (e.g., a person's intellectual ability or moral character) so that certain negative resemblances are inferred. Importantly, however, knowledge used from the original context cannot be literally true in the inferred context. For the insult lunatic, general semantic knowledge that a lunatic is someone of unsound mind (as perhaps judged from a psychiatric or legal viewpoint) can be used to infer that someone who is not really of unsound mind (from a psychiatric or legal viewpoint) nonetheless possesses certain, say, intellectual qualities or attributes that are less than desirable. For the insult asswipe, knowledge gained from certain bodily experiences can be used to infer that someone, who cannot literally be an asswipe, nonetheless possesses certain, say, moral qualities or attributes that are (to put it mildly) less than desirable. We propose that this second way of conceptualizing abstractness is highly relevant for the present study.

Thus, to be as clear as possible, we are proposing a conceptualization of abstractness in a perhaps less traditional, but nonetheless, we believe, theoretically interesting and legitimate manner. Our conceptualization of abstractness is in fact very similar to that of Lakoff and Johnson (1999) and Wilson and Gibbs (2007), described above. That is, knowledge gained from one context may be abstracted and used in another context, in which the knowledge is applied metaphorically because it cannot be applied literally. More pertinently to the question of embodiment, the negative inferences for many insults, such as asswipe, will be derived from a bodily (i.e., sensorimotor) context and applied to a different context (e.g., making implications about someone's intelligence or moral character).
The research question of interest for the present study is therefore whether this kind of knowledge gained from bodily experience influences semantic processing of insults.

We propose that if the framework of perceptual symbol systems theory were applied to processing insults, two important assumptions can be made. The first assumption is that sensory, motor, kinesthetic, and proprioceptive knowledge gained from bodily experience may be used to understand the implied meaning of some insults (i.e., more embodied insults, such as numbskull) more so than other insults (i.e., less embodied insults, such as idiot). For example, when someone is called a numbskull, comprehension of the implied meaning may comprise simulating prior experiences of numbness (e.g., in one's arms or legs), despite the fact that there is no implication of numbness literally occurring in the person's skull at the time the insult is given. Thus, for more embodied insults like numbskull, bodily knowledge (in this case of knowing what it is like to feel numbness and any resulting deficits in functionality of the associated body part) is likely to be a very salient component of their simulations. We should also emphasize here that although the majority of the more embodied insults used in our Experiments 1-3 below have body part components (as numbskull does), this does not imply that more embodied insults must have a body part component. We propose that more embodied insults, such as ugly (e.g., one may experience ugliness because of having a scrape mark on one's face) and weak (e.g., one may experience weakness because of not being able to lift a heavy object), while not including mention of body parts, also elicit greater bodily knowledge in their simulations than less embodied insults. All of these insults, whether they include mention of body parts or not, are more embodied because participants rate bodily experience as important to understanding their meanings. ${ }^{1}$

The second assumption we make is that emotional and introspective systems contain knowledge that is highly relevant to processing insults, and that these forms of knowledge are therefore involved in the simulation process. That is, although when someone is called an idiot, it is difficult to imagine what type of bodily knowledge may be simulated that would aid in comprehension, it is likely the case that this would cause negative emotional reactions (e.g., shame, anger, embarrassment), similar to when someone is called a numbskull. In addition, contextual considerations (e.g., being called an idiot or a numbskull immediately after committing a faux pas) would activate knowledge in introspective systems that would contribute to understanding that an insult has been given. In summary, our proposal is that: (a) simulating knowledge gained through emotional and introspective systems is likely to be just as important to comprehending the intentions behind more embodied insults and less embodied insults, but (b) simulating knowledge gained through sensory, motor, kinesthetic, and proprioceptive systems is likely to be much more important to comprehending the intentions behind more embodied insults than less embodied insults.

Siakaluk et al. (in press) examined the influence of knowledge gained through bodily experience on processing of insults in a variant of the emotional Stroop task. Previous research has

${ }^{1}$ Note that we expanded the item set in Experiment 4 to include many more embodied insults that did not mention a body part, and the same pattern of results was observed as in Experiments 1-3. 
found that response latencies to name the font color of emotionally laden words (e.g., taboo words such as rape) are slower than response latencies to name the font color of non-emotional words (e.g., run). It has been proposed that this emotional Stroop effect occurs because reactions (e.g., embarrassment, shock) to emotionally laden words engage processing resources needed for color naming (MacKay et al., 2004). In their variant of the emotional Stroop task, Siakaluk et al. (in press) used more embodied insults (e.g., asswipe), less embodied insults (e.g., cheapskate), and non-insults (e.g., hardwood) as stimuli. They first collected bodily experience ratings for their insult stimuli, which measured the extent to which the meaning of each insult referenced or alluded to knowledge gained through human bodily experience (see below for a more detailed explanation). In other words, the bodily experience ratings gauged how important participants thought having a body was to understanding the meanings of the insults. Siakaluk et al. (in press) then conducted multiple regression analyses on color naming latencies and reported two intriguing findings. First, there was an insult Stroop effect, such that color naming latencies were slower to the insults than to the non-insults. Second, the bodily experience ratings accounted for a significant amount of unique color naming latency variability for the insults, even after the variability attributed to other predictors (e.g., print frequency, offensiveness, number of meanings) was statistically removed. Moreover, the effect of bodily experience was inhibitory such that color naming latencies were slower for the more embodied insults than for the less embodied insults.

Siakaluk et al. (in press) proposed that the inhibitory embodied insult Stroop effect can be accommodated by Barsalou's (1999) perceptual symbol systems theory. That is, when more embodied insults are presented in the Stroop task, they activate richer simulations of knowledge gained through sensory, motor, kinesthetic, and proprioceptive systems than do less embodied insults. In other words, when participants view a more embodied insult, they simulate the bodily knowledge associated with the insult (e.g., when viewing the insult numbskull, participants simulate the knowledge of what it feels like when a body part is numb and any resulting deficits in functionality of the associated body part). These richer sensorimotor simulations that are activated by more embodied insults lead to more efficient linguistic processing, which ultimately leads to greater inhibition of color naming responses (see also Cohen et al., 1990).

\section{THE PRESENT RESEARCH}

In the present research we examined the influence of bodily knowledge in the processing of insults but in a much more direct way than in Siakaluk et al. (in press). That is, Siakaluk et al.'s Stroop study showed longer color naming latencies for relatively more embodied insults, and it was assumed that this effect arose because, for relatively more embodied insults, the insult meanings were harder to ignore (thus inhibiting color naming responding). Since the Stroop task was used in that study, however, one cannot actually be sure that participants were processing the insult meanings of the stimuli. Thus, one could assert that it is not clear that the "embodied" effects observed in Siakaluk et al. (in press) were generated in the process of deriving insult meanings. The present study addresses this issue more directly by examining the effects of bodily experience on processing of insults in a task we call the insult detection task.

There is much research demonstrating that richer semantic representations elicit faster responding in semantic categorization tasks (e.g., Pexman et al., 2008; Siakaluk et al., 2003, 2008b), of which the insult detection task is a special case (because it involves categorizing stimuli as belonging or not belonging to the decision category "insult"). It has been argued that richer semantic representations afford faster settling in the semantic units (e.g., Pexman et al., 2008) and, hence, faster semantic decision latencies. We therefore predicted a facilitatory effect for more embodied insults in the insult detection task, such that response latencies will be faster to more embodied insults than to less embodied insults. If this prediction is borne out, then it will provide support for Siakaluk et al.'s (in press) conclusion that it was indeed the activation of the meanings of the insults that inhibited Stroop task performance in their study. In the present study, richer sensorimotor simulations should provide more evidence that an item is a positive instance of the category "insult": thus, faster response latencies should be associated with more embodied insults. In contrast, in Siakaluk et al.'s (in press) Stroop task, task demands required that the participants pronounce the font color, and richer sensorimotor simulations should hinder participants' ability to pronounce the font color; thus, slower response latencies should be associated with more embodied insults, which was what was observed.

To ensure the best possible opportunity to observe facilitatory effects of bodily experience in the insult detection task, we used a go/no-go procedure (in which participants respond only to the insult stimuli), rather than a yes/no procedure (in which participants respond to both the insult and non-insult stimuli). We did this because semantic effects in semantic categorization tasks have been most robust using the go/no-go procedure, most likely because this procedure encourages more extensive processing (see, e.g., Siakaluk et al., 2003, 2007). In addition, participants completed a surprise recall task immediately after they finished the insult detection task. We included the surprise recall task in the present study in order to test an additional research question; that is, whether effects of bodily experience on insult processing are very fleeting (in which case they should not influence subsequent memory for the stimuli) or longer lasting (in which case they should influence subsequent memory for the stimuli). We predicted a facilitatory effect for more embodied insults in the surprise recall task, such that participants should recall a greater percentage of more embodied insults than less embodied insults. We made this prediction because the more detailed simulations that should be elicited for the more embodied insults should afford more elaborative processing at encoding and hence facilitate recall during the surprise recall testing phase.

\section{EXPERIMENT 1 METHOD \\ Participants}

Thirty-three undergraduate students from the University of Northern British Columbia (UNBC) participated in the experiment for bonus course credit. All were native English speakers and reported normal or corrected-to-normal vision. None of these individuals participated in any of the other experiments. 


\section{Stimuli}

Siakaluk et al. (in press) had a group of 40 UNBC undergraduate students rate 178 insults on a 1-7 Likert scale regarding how important they thought bodily experience would be to understanding the insult. The following instructions were provided to the participants to read while the researcher read them aloud:

\begin{abstract}
Please read each insult carefully and decide to what extent the meaning of the insult references or alludes to knowledge gained through human bodily experience and interactions. That is, please rate how important you think having a body contributes to understanding what each insult means.
\end{abstract}

After reading the instructions, the participants were given two examples to help them understand how to rate the insults. The researcher explained that the insult bossy would likely be given a relatively low rating because it is not clear how bodily experience would contribute much to its meaning, whereas the insult numbskull would likely be given a relatively high rating because experience with numbness in our bodies may help contribute to its intended meaning.

Thirty-six insults were selected from Siakaluk et al. (in press) for use in the present experiment: 18 of the insults (e.g., asswipe) were rated as being high in bodily experience (henceforth referred to as more embodied insults) and the other 18 insults (e.g., cheapskate) were rated as being low in bodily experience (henceforth referred to as less embodied insults). The insults consisted of either one or two words. The two groups of insults were matched on length (i.e., number of letters), print frequency (using HAL log-frequency values from the English Lexicon Project database; Balota et al., 2007), rated offensiveness (on a 1-7 scale), rated frequency of usage (on a 1-7 scale), rated number of meanings (on a 0-2 scale), and rated imageability (on a $1-7$ scale) (all ps $>0.35$ ). The descriptive statistics for the insults are presented in Table 1. Thirty-six non-insults (e.g., airbase) were selected from Siakaluk et al. (in press) to be used as control items. The non-insults also consisted of either one or two words, and were matched to the insults on printed length. The experimental stimuli are listed in the Appendix.

\section{Apparatus and procedure}

The stimulus items were presented one at a time on a computer display driven by a Pentium-class microcomputer running DirectRT software. The participants' task was to determine whether the stimulus items were insults or not. Before starting the insult detection task, the participants were primed with instructions indicating that when each stimulus item was presented they should imagine themselves in a confrontational situation where someone says to them, "You are " or "You are a(n) . A trial was initiated by a fixation marker that appeared at the center of the computer display. The fixation marker was presented for $1 \mathrm{~s}$, and was then replaced by a stimulus item. Participants were asked to respond only to the stimulus items that were insults, and to respond as quickly and accurately as possible by pressing the "?" key on the computer keyboard. If participants judged a stimulus item to not be an insult, they were asked not to make any key press response. If no response was made, stimulus items remained on the computer display for $2.5 \mathrm{~s}$, and were then removed and replaced by the fixation marker. The 72 stimuli were presented in a different random order to each participant.

Before beginning the experiment, each participant first completed 20 practice trials. The practice trials consisted of 10 insult stimuli (five more embodied insults and five less embodied insults) and 10 non-insult stimuli. All practice stimuli were similar in normative print frequency to the experimental items.

After participants completed the insult detection task, they completed a surprise recall task. Participants were given $3 \mathrm{~min}$ to recall as many stimulus items as they could remember from the insult detection task. Participants typed their responses into an Excel spreadsheet. Responses were coded as correct even if they were not spelled correctly (e.g., asswhole or wanna be were coded as correct for the insults asshole and wannabe, respectively). Synonyms, however, were not coded as correct.

\section{RESULTS AND DISCUSSION}

For all the experiments reported in the present study the following procedure for removal of outliers was used. For each participant, response latencies greater than or less than $2.5 \mathrm{SD}$ from the cell mean of each condition were treated as outliers. Across participants, this resulted in the removal of a total of 38 observations (3.20\% of the data) from the data set in Experiment 1. Response errors for the insult stimuli comprised only $2.35 \%$ of trials, and, as such, the response error data were not analyzed. The mean response latencies of correct responses, mean error percentages, and correct recall percentages for all stimulus types are presented in Table 2. For the first three experiments reported in the present study bodily experience was a within-subject manipulation, and unless otherwise indicated, all effects are significant at $p<0.05$.

There was a significant effect of bodily experience in the response latency data, $t(32)=2.68, \mathrm{SEM}=10.65$, partial $\eta^{2}=0.18$, and in the surprise recall data, $t(32)=2.54, \mathrm{SEM}=2.39$, partial $\eta^{2}=0.17$. In the response latency data, responses to the more embodied insults were an average of $28 \mathrm{~ms}$ faster than responses to the less embodied insults. In the surprise recall data, the percentage of items correctly recalled was $6.06 \%$ higher for more embodied insults than for less embodied insults.

Table 1 | Mean characteristics and standard errors (in parentheses) for the insult stimuli used in Experiments 1-3.

\begin{tabular}{|c|c|c|c|c|c|c|c|}
\hline Insult type & Bodily exp & Length & Print freq & Offensive & Usage freq & NoM & Image \\
\hline Less embodied & $2.5(0.11)$ & $6.5(0.44)$ & $6.1(0.86)$ & $3.2(0.19)$ & $3.6(0.22)$ & $1.2(0.03)$ & $3.3(0.16)$ \\
\hline
\end{tabular}

Note. Bodily exp, bodily experience rating; Length, number of letters; Print freq, print frequency using HAL log-frequency values; Offensive, rating of offensiveness; Usage freq, rated frequency of usage; NoM, rated number of meanings; Image, imageability. 
These results show faster response latencies and more accurate recall for more embodied insults as compared to less embodied insults and thus provide support for the idea that knowledge gained through bodily experience facilitates responding in a task (i.e., the insult detection task) in which richer sensorimotor simulations should be useful. Moreover, the response latency data are consistent with the facilitatory BOI effect on response latencies in semantic categorization (Siakaluk et al., 2008b; Wellsby et al., in press). The facilitatory effects of bodily experience on the surprise recall data are intriguing, as they demonstrate that bodily experience effects persist after the insult detection task is finished and are also influential in memory for insults.

One potential problem with Experiment 1 is the fact that 12 of the more embodied insults mentioned a body part, whereas only four of the non-insults mentioned a body part (if one includes the non-insult denture). It is possible that the mention of a body part in a stimulus item could have been used as a cue by participants to decide that the stimulus item was an insult. Thus, it may not have been the case that participants were creating richer sensorimotor simulations for the more embodied insults, which led to faster response latencies to these items. Instead, participants may have noticed that any stimulus item that mentioned a body part was an insult, and partly based their responses on that factor. (See above, however, for our proposal that the inclusion of a body part is not necessary for an item to be considered or judged a more embodied insult). To address this issue, we conducted Experiment 2 in which each more embodied insult that mentioned a body part (e.g., asswipe) was matched with a noninsult that also mentioned a body part (e.g., shinpad). Two outcomes were possible with this methodological change. First, if the mention of a body part was serving as an important cue in Experiment 1 that the item was an insult, then we should not find an effect of bodily experience in Experiment 2, because the mention of a body part in Experiment 2 does not reliably indicate that the item is an insult. Alternatively, if the mention of a body part was not responsible for the results in Experiment 1, but instead the more embodied insults were responded to faster than the less embodied insults because they elicited richer sensorimotor simulations, then facilitatory effects of bodily experience should again be observed in Experiment 2.

\section{EXPERIMENT 2 \\ METHOD \\ Participants}

Thirty-three UNBC undergraduate students participated in the experiment for bonus course credit. All were native English speakers and reported normal or corrected-to-normal vision. None of these individuals participated in any of the other experiments.

\section{Stimuli}

The insult stimuli were those used in Experiment 1. Twenty-four of the non-insult stimuli were the same as those used in Experiment 1 , and the remaining 12 were selected for this experiment. In this experiment there were as many non-insult stimuli that mentioned a body part (e.g., shinpad) as there were insult stimuli that mentioned a body part. The non-insult stimuli again consisted of either one or two words, and were matched as closely as possible to the insult stimuli on printed length. The new set of non-insult stimuli are listed in the Appendix.

\section{Apparatus and procedure}

The apparatus and procedure were identical to those used in Experiment 1.

\section{RESULTS AND DISCUSSION}

There were 32 observations (2.69\% of the data) treated as outliers and removed from the data set in Experiment 2. Response errors for the insult stimuli comprised only $1.30 \%$ of trials, and, as such, the response error data were not analyzed. The mean response latencies of correct responses, mean error percentages, and correct recall percentages for all stimulus types are presented in Table 2.

There was a significant effect of bodily experience in the response latency data, $t(32)=3.37, \operatorname{SEM}=9.96$, partial $\eta^{2}=0.26$, and in the surprise recall data, $t(32)=2.86, \mathrm{SEM}=2.30$, partial $\eta^{2}=0.20$. In the response latency data, responses to the more embodied insults were an average of $33 \mathrm{~ms}$ faster than responses to the less embodied insults. In the surprise recall data, the percentage of items correctly recalled was $6.56 \%$ higher for more embodied insults than for less embodied insults.

The findings that participants again responded faster to and correctly recalled more of the more embodied insults than the less embodied insults indicates that the hypothesis that mere mention of a body part in a stimulus item was used as a cue for insult categorization (and thus produced the observed facilitatory effects) in Experiment 1 can be discounted. Therefore, the results again provide support for the notion that the facilitatory effects of bodily experience are due to richer elicited sensorimotor simulations, which facilitate detection of more embodied insults during the insult detection task and also cause better encoding and later retrieval for these items.

In Experiments 1 and 2 participants had to distinguish insulting stimuli from non-insulting stimuli, and the non-insult stimuli were not selected from any one single, coherent category. It is possible that this creates a more difficult decision than a situation in which the non-insults are from one coherent category (e.g., compliments). In a similar vein, Hino et al. (2002) suggested that semantic categorization tasks vary in the extent to which they require more versus less analytic processing. Hino et al. demonstrated that by changing the decision category participants could be encouraged to engage in more versus less analytic processing. The argument here would be that, even when the decision category (insult versus non-insult) remains the same, similar modulation might be invoked as a function of the non-insults presented. By this analysis, the insult detection tasks used in Experiments 1 and 2, with heterogeneous non-insults, could have evoked relatively analytic processing to determine whether a presented stimulus is an insult or not. Presumably, richer sensorimotor simulations facilitated this relatively extensive analytical processing. The fact that we observed faster processing of more embodied insults in Experiments 1 and 2 is consistent with this analysis.

It is possible that with a more coherent set of non-insults participants could perform the insult detection task with a relatively more cursory or shallow analysis of a small set of target features. In order to test this possibility, in the present Experiment 3 we modified the non-insult stimuli so that all of them were compliments. With this more coherent set of non-insults it may be possible for participants to categorize the stimuli based on shallower processing (e.g., the 
Table 2 | Mean response latencies (in $\mathrm{ms}$ ) and standard errors, mean response error percentages and standard errors, and percentage words correctly recalled and standard errors for Experiments 1-3.

\begin{tabular}{|c|c|c|c|c|c|c|}
\hline \multirow[b]{2}{*}{ Insult type } & \multicolumn{2}{|c|}{ Experiment 1} & \multicolumn{2}{|c|}{ Experiment 2} & \multicolumn{2}{|c|}{ Experiment 3} \\
\hline & $M$ & SE & $M$ & SE & $M$ & SE \\
\hline \multicolumn{7}{|l|}{ RESPONSE LATENCIES } \\
\hline More embodied & 734 & 31.2 & 711 & 25.5 & 762 & 26.1 \\
\hline Less embodied & 762 & 37.4 & 744 & 24.4 & 809 & 31.1 \\
\hline Bodily experience effect & 28 & - & 33 & - & 47 & - \\
\hline Control items & - & - & - & - & - & - \\
\hline \multicolumn{7}{|c|}{ RESPONSE ERROR PERCENTAGES } \\
\hline More embodied & 1.71 & 0.8 & 1.41 & 0.4 & 0.36 & 0.3 \\
\hline Less embodied & 2.91 & 0.9 & 1.20 & 0.5 & 2.07 & 0.7 \\
\hline Bodily experience effect & 1.20 & - & -0.21 & - & 1.71 & - \\
\hline Control items & 3.06 & 0.7 & 3.23 & 0.8 & 3.31 & 0.7 \\
\hline \multicolumn{7}{|c|}{ PERCENTAGE WORDS CORRECTLY RECALLED } \\
\hline More embodied & 26.76 & 2.0 & 26.42 & 2.1 & 20.03 & 1.7 \\
\hline Less embodied & 20.70 & 1.6 & 19.86 & 2.0 & 14.64 & 1.6 \\
\hline Bodily experience effect & 6.06 & - & 6.56 & - & 5.39 & - \\
\hline Control items & 7.24 & 1.0 & 8.33 & 1.2 & 16.3 & 1.5 \\
\hline
\end{tabular}

emotional content of the stimuli - insults are negative, whereas compliments are positive). Therefore, in Experiment 3 we predicted two possible outcomes. First, there may not be a significant difference in response latencies between the more embodied and the less embodied insults. This null effect could arise if responses are indeed made after a relatively cursory or shallow analysis of a small set of target features, which may make the need for using simulations largely unnecessary. Second, there may be a small yet significant facilitatory effect of bodily experience. Participants may rely on a small set of target features to decide whether each stimulus is an insult or not, but simulations may still be used to make insult versus compliment decisions, which should lead to faster response latencies to the more embodied insults.

\section{EXPERIMENT 3 \\ METHOD \\ Participants}

Thirty-three UNBC undergraduate students participated in the experiment for bonus course credit. All were native English speakers and reported normal or corrected-to-normal vision. None of these individuals participated in any of the other experiments.

\section{Stimuli}

The insult stimuli were those used in Experiment 1. The non-insult stimuli consisted of compliments (e.g., hard worker, level headed), and there were an equal number of insults and compliments that mentioned a body part. The compliments consisted of either one or two words, and were matched as closely as possible to the insult stimuli on printed length. The compliment stimuli are listed in the Appendix.

\section{Apparatus and procedure}

The apparatus and procedure were identical to those used in Experiment 1.

\section{RESULTS AND DISCUSSION}

There were 36 observations (3.03\% of the data) treated as outliers and removed from the data set in Experiment 3. Response errors for the insult stimuli comprised only $1.22 \%$ of trials, and, as such, the response error data were not analyzed. The mean response latencies of correct responses, mean error percentages, and correct recall percentages for all stimulus types are presented in Table 2 .

There was a significant effect of bodily experience in the response latency data, $t(32)=3.11, \operatorname{SEM}=15.19$, partial $\eta^{2}=0.23$, and in the surprise recall data, $t(32)=2.75, \mathrm{SEM}=1.96$, partial $\eta^{2}=0.19$. In the response latency data, responses to the more embodied insults were an average of $47 \mathrm{~ms}$ faster than responses to the less embodied insults. In the surprise recall data, the percentage of items correctly recalled was $5.39 \%$ higher for more embodied insults than for less embodied insults.

We suggested above that it may be possible to induce a more cursory or shallow analysis in an insult detection task by using a coherent set of non-insults (i.e., compliments), because responses could be partially or primarily based on a small set of target features, such as emotional content. We predicted that if such was the case, then there could be an attenuated or null effect of bodily experience in Experiment 3. However, the results for Experiment 3 are clear: bodily experience again facilitated responding and recall.

The findings from Experiments 1-3 are consistent with the idea that sensorimotor simulations were an important component of how insults were processed, regardless of the type of non-insults used. First, the effect size for the response latency data of the present experiment (partial $\eta^{2}=0.23$ ) is comparable to the effect sizes for the response latency data in Experiments 1 and 2 (partial $\eta^{2}=0.18$ and partial $\eta^{2}=0.26$, respectively). Second, the effect size for the recall data of the present experiment (partial $\eta^{2}=0.19$ ) is comparable to the effect sizes for the recall data in Experiments 1 and 2 (partial $\eta^{2}=0.17$ and partial $\eta^{2}=0.20$, respectively). 
One potential criticism of the previous three experiments is that they used a relatively small stimulus set (even though the more embodied and less embodied insults were carefully matched on numerous confound variables). In order to address this potential criticism, Experiment 4 was designed to investigate whether the facilitatory effect of bodily experience observed in Experiments 1-3 could be extended to a larger set of items. To do this, we conducted another insult detection task, identical in procedure to the previous experiments, but consisting of a much larger number of items (from Siakaluk et al., in press). In this new stimulus set the more embodied insults and less embodied insults were not matched on any of the variables that were controlled in the previous experiments; instead, we controlled these variables statistically, using hierarchical multiple regression analyses for the response latency, response error, and recall data. On the first step of each analysis we entered the control variables of printed length, morphological complexity, HAL log-frequency, and frequency of usage, number of meanings, offensiveness, and imageability ratings as the predictors, and on the second step we entered bodily experience ratings as the predictor. We predicted that bodily experience ratings would account for a significant amount of unique response latency, response error, and recall variability, above and beyond the variability accounted for by the control variables.

\section{EXPERIMENT 4 METHOD \\ Participants}

Thirty-seven UNBC undergraduate students participated in the experiment for bonus course credit. All were native English speakers and reported normal or corrected-to-normal vision. None of these individuals participated in any of the other experiments. Data for seven of these participants were excluded from the analyses because they had error rates of more than $25 \%$ to the insult stimuli.

\section{Stimuli}

The full stimulus set from Siakaluk et al. (in press) was used in this experiment. Therefore, there were 178 insults ( 88 more embodied insults and 90 less embodied insults), and 179 non-insults. The experimental stimuli are available from the corresponding author.

\section{Apparatus and procedure}

The same procedure used in Experiments 1-3 was also used in Experiment 4, with the following exception: here participants were allowed to take a break after every 90 trials (the last block of trials always had 88 trials). The stimuli were presented in a different random order to each participant.

\section{RESULTS AND DISCUSSION}

Data for 19 of the insult stimuli (12 more embodied insults and 7 less embodied insults) were excluded from the analyses because response error rates for those items were greater than 25\%. There were 145 observations (3.29\% of the data) treated as response latency outliers and removed from the data set in Experiment 4.

Hierarchical multiple regression analyses were conducted to examine whether significant amounts of unique response latency, response error, and recall variability could be accounted for by bodily experience ratings. As noted, for each analysis, the control variables entered in step one were printed length, morphological complexity, HAL log-frequency, and ratings of frequency of usage, number of meanings, offensiveness, and imageability. Bodily experience ratings were entered in step two. Zero-order correlations between the predictor variables and the criterion variables are shown in Table 3. The multiple regression results for the response latency, response error, and recall data are presented in Tables 4, 5, and 6, respectively. Of most importance for the present study is the fact that the hypothesis that the bodily experience ratings would account for a significant amount of unique response latency, response error, and recall variability, above and beyond the variability accounted for by the control variables, was supported. Two statistically significant results are of immediate interest. First, for the response latency and error analyses the semi-partial correlation between bodily experience ratings and both criterion variables was negative, indicating that responses to relatively more embodied insults were faster and more accurate. Second, for the recall analysis the semi-partial correlation between bodily experience and percent recall was positive, indicating that greater recall occurred for relatively more embodied insults. In summary, the results from the present experiment replicate and extend the results from the first three experiments to a much larger stimulus set.

Table 3 | Zero-order correlations between the predictor variables and the criterion variables in Experiment 4.

\begin{tabular}{|c|c|c|c|c|c|c|c|c|c|c|c|}
\hline Measure & 1 & 2 & 3 & 4 & 5 & 6 & 7 & 8 & 9 & 10 & 11 \\
\hline 1. IDT response latency & - & & & & & & & & & & \\
\hline 2. IDT response error rate & $0.47^{* *}$ & - & & & & & & & & & \\
\hline 4. Printed length & $0.49 * *$ & $0.18^{*}$ & -0.12 & - & & & & & & & \\
\hline 5. Morphological complexity & $0.37^{* *}$ & 0.11 & -0.04 & $0.73^{* *}$ & - & & & & & & \\
\hline 8. Number of meanings & -0.10 & -0.07 & 0.15 & $-0.16^{*}$ & -0.13 & $0.38 * *$ & $0.28 * *$ & - & & & \\
\hline 9. Offensiveness ratings & $-0.24^{* *}$ & $-0.43^{* *}$ & $0.18^{*}$ & 0.10 & 0.04 & 0.08 & $0.37^{* *}$ & 0.13 & - & & \\
\hline 10. Imageability ratings & 0.09 & $0.16^{*}$ & $0.24 * *$ & -0.09 & -0.05 & 0.04 & 0.10 & $0.43^{* *}$ & -0.02 & - & \\
\hline 11. Bodily experience ratings & $0.18 *$ & 0.03 & $0.17^{*}$ & $0.35 * *$ & $0.36 * *$ & 0.04 & $-0.31 * *$ & -0.02 & -0.02 & $0.31 * *$ & - \\
\hline
\end{tabular}

Note. IDT, insult detection task; HAL, hyperspace analog to language.

${ }^{*} p<0.05,{ }^{*} p<0.01$. 
Table 4 | Results of hierarchical multiple regression analysis for insult detection task response latency data for Experiment 4.

\begin{tabular}{|c|c|c|c|c|c|c|}
\hline Variable & $B$ & SEB & $\boldsymbol{\beta}$ & sr & $R^{2}$ & $\Delta R^{2}$ \\
\hline Step 1 (control variables) & & & & & $0.446 * * *$ & \\
\hline Step 2 & & & & & $0.487^{* * *}$ & $0.021^{*}$ \\
\hline Printed length & 16.94 & 4.01 & 0.39 & $0.25^{* * *}$ & & \\
\hline Morphological complexity & -0.61 & 14.81 & -0.01 & -0.00 & & \\
\hline Number of meanings & 2.55 & 45.84 & 0.00 & 0.00 & & \\
\hline Offensiveness ratings & -13.35 & 8.48 & -0.10 & -0.09 & & \\
\hline Imageability ratings & 24.07 & 7.68 & 0.22 & $0.18^{* *}$ & & \\
\hline Bodily experience ratings & -12.73 & 5.20 & -0.17 & $-0.14^{*}$ & & \\
\hline
\end{tabular}

Note. HAL, hyperspace analog to language. The $B, S E B, \beta$, and sr values are for the final step in the analysis, where all the predictor variables were included in the equation. ${ }^{*} p<0.05,{ }^{* *} p<0.01,{ }^{* *} p<0.001$

Table 5 | Results of hierarchical multiple regression analysis for insult detection task response error data for Experiment 4.

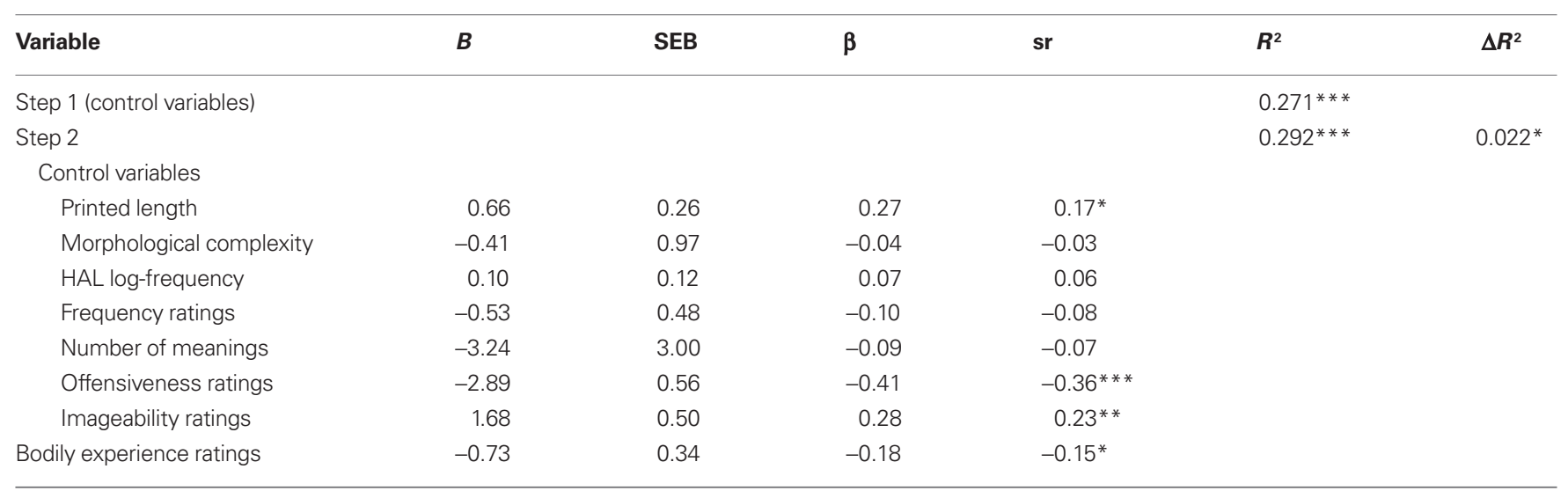

Note. HAL, hyperspace analog to language. The B, SEB, $\beta$, and sr values are for the final step in the analysis, where all the predictor variables were included in the equation. ${ }^{*} p<0.05,{ }^{*} p<0.01,{ }^{* *} p<0.001$

Table 6 | Results of hierarchical multiple regression analysis for insult detection task word recall data for Experiment 4.

\begin{tabular}{|c|c|c|c|c|c|c|}
\hline Variable & $B$ & SEB & $\beta$ & sr & $R^{2}$ & $\Delta R^{2}$ \\
\hline Step 1 (control variables) & & & & & $0.174 * *$ & \\
\hline Step 2 & & & & & $0.220 * *$ & $0.046^{*}$ \\
\hline Printed length & -0.54 & 0.48 & -0.13 & -0.08 & & \\
\hline Morphological complexity & 1.55 & 1.77 & 0.10 & 0.06 & & \\
\hline Number of meanings & -2.05 & 5.49 & -0.03 & -0.03 & & \\
\hline Offensiveness ratings & 0.73 & 1.02 & 0.06 & 0.05 & & \\
\hline Imageability ratings & 1.31 & 0.92 & 0.13 & 0.10 & & \\
\hline Bodily experience ratings & 1.86 & 0.62 & 0.26 & $0.22^{*}$ & & \\
\hline
\end{tabular}

Note. HAL, hyperspace analog to language. The B, SEB, $\beta$, and sr values are for the final step in the analysis, where all the predictor variables were included in the equation. ${ }^{*} p<0.01,{ }^{*} p<0.001$ 


\section{GENERAL DISCUSSION}

In the present research we used a task called an insult detection task (a type of semantic categorization task) to examine the influence of bodily knowledge on insult processing. In previous research it has been reported that richer semantic representations lead to faster responding in semantic categorization tasks (e.g., Siakaluk et al., 2003, 2008b; Pexman et al., 2008), and by this logic, we predicted that because more embodied insults should elicit richer sensorimotor simulations (i.e., representations), responses would be faster to more embodied insults as compared to less embodied insults. As predicted, a facilitatory effect for more embodied insults was observed in the response latency data for each of the insult detection tasks reported in the present research (as well as in the response error data for Experiment 4). As such, the results from the present research support the inference that knowledge gained through bodily experience plays an important role in the detection of insults.

After each experiment we conducted a surprise recall task. We predicted that participants would recall a greater percentage of more embodied insults than less embodied insults, because the former type of insult would elicit richer sensorimotor simulations, which should aid in their subsequent recall. As predicted, a greater percentage of more embodied insults were correctly recalled in each of the four experiments. The recall results demonstrate that there are facilitatory effects of bodily experience that are still present after completion of the insult detection task. As such, the embodied effects we observed in the insult detection task are not fleeting but rather influence behavior subsequent to the detection task.

As noted in the Introduction, a theory that can be used to explain the facilitatory effects of bodily experience in insult detection and recall performance is Barsalou's (1999) perceptual symbol systems theory. By this account, bodily interactions with the environment are crucial to many cognitive processes. Barsalou (1999) suggested that the modality-specific neural systems used for perception, action, and introspection are also used to represent concepts in the brain, through the process of simulation. According to Barsalou (1999, 2008), simulation is the partial neural re-enactment of perceptual, motor, and introspective states acquired during bodily experience with the environment. When we conceptualize an object or event, what is occurring is that we are neurally re-experiencing the sensory, motor, and introspective components associated with that object or event from previous bodily experience.

The results of the present study are consistent with the idea that more embodied insults are at least partly understood by creating simulations of bodily experience. As noted above, while simulations for emotional knowledge (e.g., negative emotions evoked by insults) and introspective knowledge (e.g., contextual or situational knowledge gained from previous experiences using or being called a particular insult) would likely be equally rich for more embodied and less embodied insults, the two types of insults differ in the amount of knowledge that is gained through bodily (i.e., sensory, motor, kinesthetic, and proprioceptive) experience. For example, we may simulate the sensation of numbness when we comprehend the insult numbskull. Therefore, because of knowledge based on bodily experience, more embodied insults elicit richer sensorimotor simulations (i.e., greater neural re-enactment of perceptual, motor, kinesthetic, and proprioceptive states; Barsalou, 1999, 2008) than less embodied insults. As a result, as demonstrated in the present research, richer sensorimotor simulations lead to faster insult detection latencies. Barsalou's $(1999,2008)$ notion of simulation can also be used to explain the recall findings. The richer simulations based on knowledge gained through previous bodily experience that were elicited for the more embodied insults during encoding (i.e., when they were being processed during the insult detection task) were more easily reactivated during retrieval (i.e., during the time period allotted to participants to recall as many stimuli as they could), resulting in a greater percentage of more embodied insults being recalled.

In Experiments 2 and 3 of the present research we were able to discount hypotheses suggesting that simulations may not be playing a crucial role in insult processing. One potential issue with Experiment 1 was that 12 of the more embodied insults mentioned a body part, whereas only four of the non-insults mentioned a body part. We therefore hypothesized that perhaps the mention of a body part in a stimulus item could have been used as a cue by participants that the stimulus item was an insult. That is, it may not have been the case that participants were creating richer sensorimotor simulations for the more embodied insults, which led to faster response latencies to these items. Instead, the participants may have noticed that any stimulus with mention of a body part was an insult, and partly based their responses on that factor. To address this issue, Experiment 2 was conducted, in which each more embodied insult that mentioned a body part (e.g., asswipe) was matched with a non-insult that also mentioned a body part (e.g., shinpad). The results indicated that participants again responded faster to the more embodied insults and recalled a greater percentage of more embodied insults, indicating that the hypothesis that the mention of a body part in a stimulus item was underlying the observed facilitatory effect in Experiment 1 could be discounted. Therefore, the results from Experiment 2 again provided support for the notion that the facilitatory effects of bodily experience are due to richer elicited sensorimotor simulations for the more embodied insults.

In Experiment 3 we examined the possibility that with a more coherent set of non-insults participants could perform the insult detection task with a relatively more cursory or shallow analysis of a small set of target features, thus rendering simulations unnecessary. To examine this possibility, all the non-insults used in Experiment 3 belonged to the same category (i.e., compliments) as opposed to the more general sets of non-insults used in the first two experiments. The results indicated that under conditions in which a well-defined decision category was used (i.e., insults versus compliments) participants still responded faster to and recalled more of the more embodied insults than the less embodied insults, which replicated what was observed in Experiments 1 and 2. Effect sizes (presented in the Results section of Experiment 3 ) indicated that there was no difference in the magnitude of the bodily experience effect between Experiments 1,2, and 3. Therefore, it seems likely that simulations were underlying responding in all three experiments, and were not modulated by the type of non-insult used.

The present finding of a facilitatory effect of bodily experience on insult detection (and recall) contrasts with the findings of Siakaluk et al. (in press), who examined the effects of bodily experience in a version of the Stroop task. As noted, Siakaluk et al. (in press) reported an inhibitory bodily experience effect in the Stroop 
task such that color naming latencies were slower to relatively more embodied insults. As noted above, the nature of the effect that bodily experience has on performance (i.e., either a facilitatory or an inhibitory effect) appears to be a function of task demands. In the present study, richer sensorimotor simulations provide more evidence that an item is a positive instance of the category "insult." In contrast, in Siakaluk et al.'s (in press) insult Stroop task, task demands required that the participants pronounce the font color. Thus, in the Stroop task, richer sensorimotor simulations hinder participants' ability to pronounce the font color, which leads to slower color naming latencies for the more embodied insults.

The results of the present study extend the findings of Siakaluk et al. (in press) in two important ways. First, as noted above, although Siakaluk et al. (in press) assumed that it was the richer meanings of the more embodied insults that inhibited Stroop task performance, the Stroop task is not a direct measure of the influence of word meaning. The insult detection tasks used in the present study address and resolve this concern because the task more directly assesses the processing of insult meanings. Since the predicted facilitatory effect of bodily experience was observed in a task requiring the processing of insults, we can be more confident that it was indeed the processing of insult meaning that lead to the slowing of Stroop task performance in Siakaluk et al.'s (in press) study. Second, the fact that bodily experience has been observed to both facilitate and inhibit responding (depending on the task and its demands) suggests that simulation is not a process that is simply invoked when convenient, or when it might help participants to be more efficient in a particular task. Rather, the fact that the bodily experience dimension can have both positive and negative consequences for performance suggests that it is an important, non-optional aspect of processing insults.

\section{CONCLUSION}

One of the aims of this special issue is to provide an account for the way abstract concepts are represented. The present research has taken a step toward this goal by providing new understanding

\section{REFERENCES}

Balota, D. A., Yap, M. J., Cortese, M. J., Hutchison, K. A., Kessler, B., Loftis, B., Neely, J. H., Nelson, D. L., Simpson, G. B., and Treiman, R. (2007). The English lexicon project. Behav. Res. Methods 39, 445-459.

Barsalou, L. W. (1999). Perceptual symbol systems. Behav. Brain Sci. 22, 577-660.

Barsalou, L. W. (2008). Grounded cognition. Annu. Rev. Psychol. 59, 617-645.

Cohen, J. D., Dunbar, K., and McClelland, J. (1990). On the control of automatic processes: a parallel distributed processing account of the Stroop effect. Psychol. Rev. 97, 332-361.

Cowart, M. (2004). Embodied cognition. Internet Encyclopedia of Philosophy, http://wwww.iep.utm.edu/.

about how insults are processed. One can easily imagine a situation in which someone did not act with intelligence and is called a numbskull. In this situation, one immediately understands two things. First, the term numbskull is meant as an insult, and, second, the insult is not based on the individual's skull literally being in a state of numbness. How, then, is it possible to understand the seemingly abstract meaning of numbskull?

We proposed above that there are at least two ways an insult can be thought of as abstract. First, many insults (e.g., stupid) express a quality or attribute that is distinct from any particular person. This conceptualization of abstractness seems appropriate for many of the insults in Experiment 4, which fit the sentence, "You are ___. However, many other insults do not seem appropriate for this conceptualization of abstractness. A second way of conceptualizing the abstractness of insults is that they require metaphorical understanding. That is, they require knowledge gained from one context to be applied to a different context. In the specific case of the insult numbskull, comprehension requires that knowledge gained from the context of bodily experience be applied to a different context, such as one's intellectual ability (e.g., You are a numbskull). In this, and many other cases, it is likely that the intended insult meanings are understood metaphorically, because knowledge used from bodily experience cannot literally be true in the context of one's intellectual ability (i.e., it is not the case that the person to whom the insult is directed literally has a skull that is numb). This conceptualization of abstractness is likely to be appropriate for many of the insults in Experiments $1-3$, of which the majority fit the sentence, "You are a(n) ___," although it is likely to be relevant for many of the insults used in Experiment 4 as well. Finally, we have proposed that Barsalou's $(1999,2008)$ perceptual symbols systems theory provides an elegant account of how simulation, or neural re-enactment, grounds abstract meanings of insults in modality-specific neural systems used for perception, action, and introspection. In this way, we have extended grounded cognition to a new context; that is, comprehension of insults.

J. R. (2004). Relations between emotion, memory, and attention: evidence from taboo Stroop, lexical decision, and immediate memory tasks. Mem. Cognit. 32, 474-488.

Myung, J.-Y., Blumstein, S. E., and Sedivy, J.C. (2006). Playing on the typewriter, typing on the piano: manipulation knowledge of objects. Cognition 98, 223-243.

Neisser, J. (2003). The swaying form: imagination, metaphor, embodiment. Phenomenol. Cogn. Sci. 2, 27-53.

Pexman, P. M., Hargreaves, I. S., Siakaluk, P.D., Bodner, G. E., and Pope, J. (2008). There are many ways to be rich: effects of three measures of semantic richness on visual word recognition. Psychon. Bull. Rev. 15, 161-167.

Siakaluk, P. D., Buchanan, L., and Westbury, C. (2003). The effect of semantic distance in yes/no and go/ no-go semantic categorization tasks. Mem. Cognit. 31, 100-113.

Siakaluk, P.D., Pexman, P. M., Aguilera, L., Owen, W. J., and Sears, C. R. (2008a). Evidence for the activation of sensorimotor information during visual word recognition: the body-object interaction effect. Cognition 106, 433-443.

Siakaluk, P. D., Pexman, P. M., Sears, C. R., Wilson, K., Locheed, K., and Owen, W. J. (2008b). The benefits of sensorimotor knowledge: body-object interaction facilitates semantic processing. Cogn. Sci. 32, 591-605.

Siakaluk, P. D., Pexman, P. M., Dalrymple, H.-A. R., Stearns, J., and Owen, W. J. (in press). Some insults are more difficult to ignore: the embodied insult Stroop effect. Lang. Cogn. Process.

Siakaluk, P. D., Pexman, P. M., Sears, C., and Owen, W. (2007). Multiple mean- 
ings are not necessarily a disadvantage in semantic processing: evidence from homophone effects in semantic categorisation. Lang. Cogn. Process. 22, 453-467.

Tillotson, S. M., Siakaluk, P. D., and Pexman, P. M. (2008). Body-object interaction ratings for 1,618 monosyllabic nouns. Behav. Res. Methods 40, 1075-1078.

Wellsby, M., Siakaluk, P. D., Owen, W. J., and Pexman, P. M. (in press). Embodied semantic processing: the body-object interaction effect in a non-manual task. Lang. Cogn.

Wilson, M. (2002). Six views of embodied cognition. Psychon. Bull. Rev. 9, 625-636.

Wilson, N. L., and Gibbs, R. W. (2007). Real and imagined body movement primes metaphor comprehension. Cogn. Sci. 31, 721-731.

Yaxley, R. H., and Zwaan, R. A. (2007). Simulating visibility during language comprehension. Cognition 150, 229-236.
Conflict of Interest Statement: The authors declare that the research was conducted in the absence of any commercial or financial relationships that could be construed as a potential conflict of interest.

Received: 14 July 2010; accepted: 22 October 2010; published online: 16 November 2010.

Citation: Wellsby $M$, Siakaluk PD, Pexman PM and Owen WJ (2010) Some insults are easier to detect: the embodied insult detection effect.
Front. Psychology 1:198. doi: 10.3389/ fpsyg.2010.00198

This article was submitted to Frontiers in Cognition, a specialty of Frontiers in Psychology.

Copyright (C) 2010Wellsby, Siakaluk, Pexman and Owen. This is an open-access article subject to an exclusive license agreement between the authors and the Frontiers Research Foundation, which permits unrestricted use, distribution, and reproduction in any medium, provided the original authors and source are credited. 


\section{APPENDIX \\ STIMULI USED IN EXPERIMENTS 1-3 \\ More embodied insults}

airhead, asshole, asswipe, brown noser, butt head, crybaby, dick head, dink, douchebag, dumbass, jackass, jerk off, loud mouth, pinhead, potty mouth, prick, ugly, weak

\section{Less embodied insults}

bastard, bugger, cheapskate, cheater, crazy, disgrace, dork, drama queen, ignorant, immature, lunatic, moron, nerd, pansy, retard, stupid, twit, wannabe

\section{Non-insult stimuli used in Experiment 1}

airbase, armband, ashtray, ball cap, bandage, burlap, capsize, carve, chandelier, climate, clock work, darn, dashboard, day dream, denture, diameter, disc, gas station, immunize, intrigue, jawbone, lottery, mocha, node, plant pot, polka, pullout, purse, ribbed, simple life, sizzle, summer love, tale, undo, weekend, wife

\section{Non-insult stimuli used in Experiment 2}

armband, ball cap, bandage, blue eyes, burlap, capsize, carve, chandelier, climate, daisy, darn, dashboard, diameter, foot, fun, haircut, immunize, intrigue, jawbone, kidneys, knee brace, lottery, mocha, node, nose hair, polka, purse, ribbed, rough hands, shinpad, sizzle, streaky, summer love, tale, toenail, undo, utensil, weekend, white teeth, wife

\section{Compliments used in Experiment 3}

agile, amazing, angel, awesome, ballsy, brainy, brave, bright eyed, clear skinned, cute, dependable, dreamy, eyeful, flexible, fragrant, friendly, funny, generous, genius, gutsy, handy, hardcore, hard worker, helpful, honest, level headed, limber, lovable, mindful, muscular, patient, sexy, sharp eyed, slender, smooth, sociable, soulful, strong, stylish, wise 